\title{
Mandibular-Fracture Repair in Monkeys
}

\author{
ROBERT A. BRUCE, GERALD H. BONNETTE, and JAMES R. HAYWARD \\ University of Michigan School of Dentistry, Department of Oral Surgery \\ Ann Arbor, Michigan 48104
}

\begin{abstract}
Studies of technical variables associated with mandibular-fracture in 60 monkeys have shown fracture union to be dependent on the type of surgical fracture and methods of immobilization. The age of the monkey, prolonged immobilization, and intraoral or extraoral surgical procedures had little effect on the number of unions.
\end{abstract}

Experimental studies on repair of mandibular fractures in large animals have been limited, often by a lack of standardized and reproducible models and methods. Several authors studying methods and technics for mandibular-fracture immobilization in dogs have noted that intermaxillary fixation is not necessary for fracture healing. ${ }^{1-6}$ Conversely, other investigators have observed a high degree of nonunion and infection under similar experimental conditions. ${ }^{7,8}$ Because of the limited numbers of experimental animals used, conflicting results also have been noted by authors studying mandibular-fracture repair in rhesus monkeys. . $^{9-12}$

The purpose of this investigation was to develop standardized methodology to study the many variables associated with mandibular-fracture repair. Studies were designed to evaluate the effect on healing of intraoral and extraoral surgical procedures, immobilization technics, and methods of fracture production in monkeys.

\section{Materials and Methods}

EXPERIMENTAL DESIGN.-Investigations were carried out on 60 rhesus monkeys (Macaca mulatta) weighing 2 to $4 \mathrm{~kg}$. The monkeys were divided into three groups of 20 monkeys each (Table).

Twenty monkeys approximately 3 years

This investigation was supported by the US Army Medical Research and Development Command Contract No. DA-49-193-MD-2586, Washington, DC 20360.

Received for publication March 12, 1969. old (group 1) were used to compare intraoral and extraoral surgical procedures after six weeks. A second group of 20 monkeys, 3 years old (group 2), was used to evaluate methods and duration of jaw immobilization and their effects on fracture repair. A presurgically constructed acrylic appliance was used for fracture immobilization in half of the monkeys, and interdental wiring with applied cold-curing acrylic resin was used in the other ten monkeys in group 2. Half the monkeys were killed at six and half at ten weeks after surgery.

A different method of fracture production and the effect of the monkey's age on fracture healing were studied in group 3 . The monkeys were approximately 3 and 5 years of age, and two types of induced mandibular fractures, described as technical procedures, were evaluated.

All monkeys in the study were fed a commercial soft diet* ad libitum and in all instances were able to maintain or gain weight beyond their preoperative values. The monkeys were given $100 \mathrm{mg}$ of tetracycline hydrochloride intramuscularly, one week before killing in order to determine calcification across the fracture site by use of fluorescent technics. The monkeys were killed by a barbiturate overdose, and the mandible disarticulated and placed in a $10 \%$ neutral buffered formalin solution.

TECHNICAL PROCEDURES.-Dissociative anesthesia was obtained by the use of intramuscular phenylcyclidine hydrochloride in a dosage of $2 \mathrm{mg} / \mathrm{kg}$ body weight. The monkeys in group 1 were 3 years of age, as determined by dental development. ${ }^{13}$ The mandibular right second deciduous molar and developing second premolar were extracted, and after two weeks of healing, a

* $\mathrm{R}_{\mathrm{X}}$ for Primates, Cooperative GLF Exchange, Inc, Ithaca, NY. 
TABLE 1

EFFect of Treatment Variables on Mandibular-Fracture REPAIR iN RHESUS MONKEYS

\begin{tabular}{|c|c|c|}
\hline Variable & $\begin{array}{c}\text { Animals with } \\
\text { Fracture Union }\end{array}$ & $\begin{array}{c}\text { Animals with } \\
\text { Fracture Nonunion }\end{array}$ \\
\hline \multicolumn{3}{|l|}{ Group 1: } \\
\hline \multicolumn{3}{|c|}{$\begin{array}{l}\text { Surgical fracture immobilized with } \\
\text { transosseous wiring } 6 \text { weeks }\end{array}$} \\
\hline Intraoral surgical procedure & 6 & 4 \\
\hline Extraoral surgical procedure & 5 & 5 \\
\hline \multicolumn{3}{|c|}{ Group 2 (Extraoral surgical procedure): } \\
\hline \multicolumn{3}{|c|}{$\begin{array}{l}\text { Surgical fracture through buccal and lingual } \\
\text { plates with preconstructed appliance }\end{array}$} \\
\hline Immobilized 6 weeks & 3 & 2 \\
\hline Immobilized 10 weeks & 2 & 3 \\
\hline \multicolumn{3}{|l|}{$\begin{array}{l}\text { Surgical defect immobilized with } \\
\text { interdental wiring and acrylic resin }\end{array}$} \\
\hline Immobilized 6 weeks & 4 & 1 \\
\hline Immobilized 10 weeks & 3 & 2 \\
\hline \multicolumn{3}{|c|}{$\begin{array}{l}\text { Group } 3 \text { (Extraoral surgical procedure, } \\
\text { buccal cortex only): }\end{array}$} \\
\hline \multicolumn{3}{|c|}{$\begin{array}{l}\text { Surgical fracture immobilized } 6 \text { weeks with } \\
\text { interdental wiring and acrylic resin }\end{array}$} \\
\hline 3-year-old monkeys & 10 & 0 \\
\hline 5-year-old monkeys & 9 & 1 \\
\hline
\end{tabular}

surgical fracture was created through the extraction site.

Ten monkeys were operated on through an extraoral surgical approach. The right mandible was exposed through a $3-\mathrm{cm}$ incision below the lower border. The periosteum was reflected from the buccal and lingual surface, and a vertical cut was made through the previous extraction site with a no. 6 round bur.

In the remaining ten monkeys of group 1 , the fracture was created through an intraoral surgical approach. A large mucoperiosteal flap was reflected from the buccal surface of the mandible, and a surgical cut made through the entire thickness of the mandible with a no. 6 dental bur.

In group 1, a 24-gauge stainless transsosseous wire was placed through the mandible for fixation of the fragments. Although the fragments were immobilized initially, with function, they soon became mobile. Other methods were subsequently used in groups 2 and 3.

All monkeys in group 2 were operated on through an extraoral approach to produce the surgical fracture. In ten monkeys, impressions had been taken of the mandibular teeth, and a splint had been preconstructed of clear, cold-curing acrylic resin* (Fig 1). After the surgical fracture was produced, the splint was wired into position.

In the other ten monkeys of group 2, a 24-gauge wire was twisted around the mandibular teeth adjacent to the fracture site. The long ends of the wires were twisted and bent along the buccal surfaces of the teeth across the fracture (Fig 2). A coldcuring acrylic resin $\dagger$ was incorporated around the wires and buccal surfaces of the teeth with a brush (Fig 3). Five monkeys with the preconstructed acrylic splint and five monkeys with the interdental wiring and quick-setting resin splints were killed six weeks after surgery, and the remaining ten monkeys were allowed to heal ten weeks before killing.

In group 3, ten monkeys were 3 years old, and ten were 5 years old. All fractures were produced through the extraoral approach; however, the surgical cut was made through the buccal cortex only. The fracture of the remaining lingual plate was completed by a twist of an elevator, and mobility was demonstrated manually. This re-

\footnotetext{
* Hygienic Perm, Hygienic Dental Manufacturing Co., Akron, Ohio.

$\uparrow$ Lura Lay Inlay Pattern Resin, Reliance Dental Manufacturing Co., Chicago, Ill.
} 
sulted in a true fracture of the lingual cortex, rather than a surgical defect through both cortical plates. Fracture immobilization was maintained by interdental wiring and applied acrylic resin. All monkeys in this group were killed six weeks later.

EVALUATION OF FRACTURE REPAIR.-Radiographs, manipulation, light microscopy, and ultraviolet-light microscopy noting tetracycline labeling were used. After killing, splints and wires were removed, and a man-

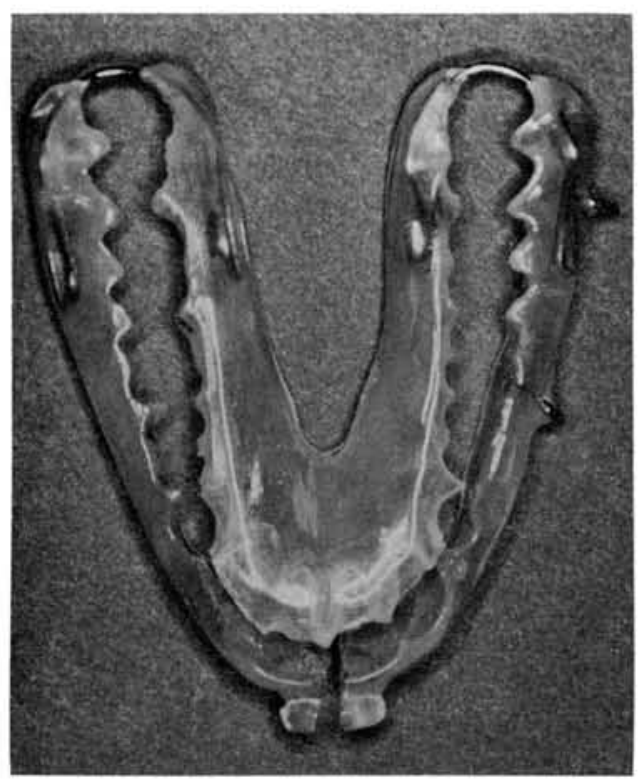

FIG 1.-Acrylic splint constructed presurgically and used for stabilization and fixation of the fractured mandible.

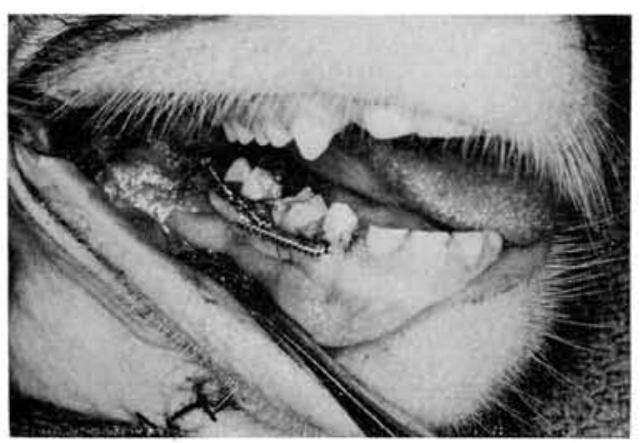

FIG 2.-Reduced fracture with wires twisted about teeth adjacent to the fracture site, then twisted together with ends bent along the buccal surfaces before incorporation with acrylic material.

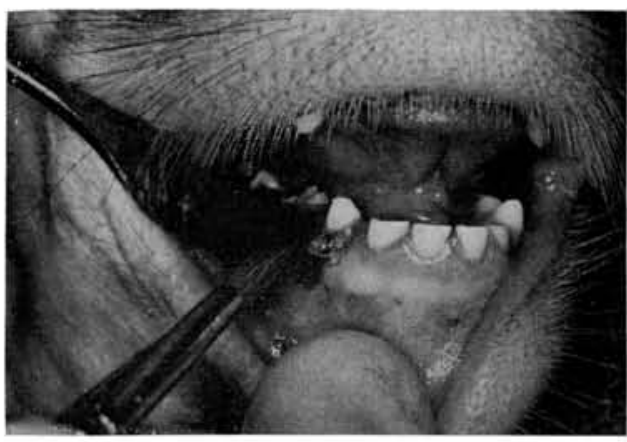

FIG 3.-Application of acrylic material about the teeth, wires, and alveolar segment with a brush.

ual evaluation of the presence or absence of fracture mobility was determined.

Radiographic evaluation required a series of lateral jaw views, preoperatively, immediately after fracture fixation, and at the time of killing (Fig 4). Also, a radiograph was obtained of the thin, calcified ground section of the fracture made parallel to the inferior border that was used in the ultraviolet-fluorescence-microscopy (Fig 5). The lateral jaw films were taken at $65 \mathrm{kv}$ and

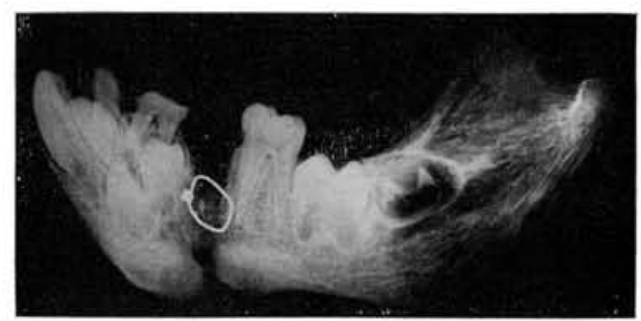

FIG 4.-Postmortem radiograph of an ununited fracture in a 3-year-old monkey, which was operated by use of an intraoral surgical procedure. Note the transosseous wire used for fixation of the bony fragments.

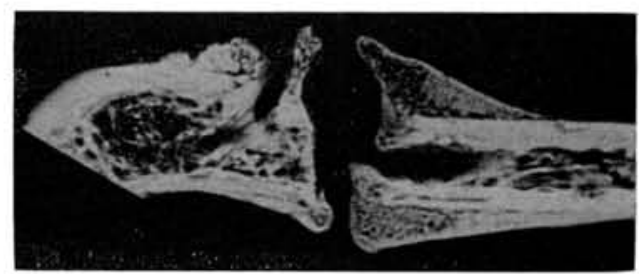

FIG 5.-Radiograph of undecalcified, ground section used in ultraviolet-fluorescence microscopy. This was typical of the "nonunion" fractures. 
60 seconds on fine-grain, industrial radiographic film."

Tissues were prepared for histologic and ultraviolet-fluorescence microscopy by sectioning in a standard horizontal plane through the fracture line parallel to the inferior border. A horizontal section of bone approximately $1 \mathrm{~mm}$ thick was taken at a level $5 \mathrm{~mm}$ above the inferior border of the mandible. These thin, ground-bone sections were radiographed as previously described and were prepared for ultravioletmicroscopic observation of tetracycline fluorescence by processing through progressive alcohol dehydration, infiltration, embedding in a clear, hard-setting plastic, polishing to approximately 20 micrometers $(\mu \mathrm{m})$, and mounting, unstained.

The remaining inferior and superior segments of the specimen not used for the ground section were decalcified, embedded, sectioned, and stained with hematoxylin and eosin for light-microscopic analysis.

A complete fracture "union" was classified when there was manual rigidity at the fracture site with radiographic and histologic evidence of bony bridging. A fracture was classified a "nonunion" when there was clinical evidence of mobility and there was no radiographic or histologic evidence of bony bridging across the fracture site.

\section{Results}

Results are presented in the Table. Consistency was noted in the four methods to evaluate fracture healing. A fracture that demonstrated rigidity with manipulation six weeks after surgery showed evidence of radiographic density and fluorescence across the fracture site. In the fractures classified as "union," after six weeks of healing, bone bridging was noted at the fracture site (Fig 6). Conversely, "nonunion" fractures demonstrated mobility, radiolucency between the fracture fragments, lack of fluorescence across the fracture site, and only fibrous connective tissue between the bony fragments without bone bridges.

Of the evaluation technics, the histologic study proved to be the most discriminating. Manipulation of the fragments was subjective, and radiographic technics were difficult to measure objectively, since calcification occurred in a random fashion throughout the fracture site. Tetracycline fluorescence

* Eastman Kodak, Rochester, NY,

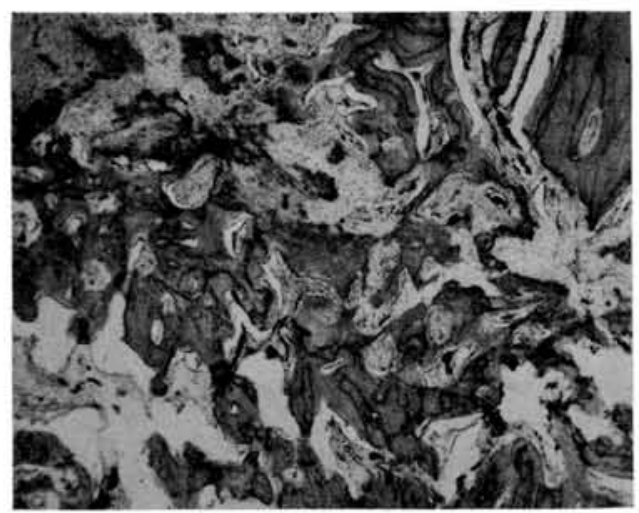

FIG 6.-Histologic section of a "union" fracture. The fibrous bone bridging the fracture was common after six weeks of healing (orig mag $\times 40)$.

showed areas of calcification, but the technic added little to the standard histologic findings.

On the basis of this limited sample, there was little difference in healing between the monkeys operated on from an intraoral approach, when compared with the extraoral approach. Fracture production was technically simplified in the extraoral approach, and, therefore, it was selected for 50 of the 60 monkeys used in other aspects of the study.

Intraosseous wires used to hold the fragments without supplemental dental fixation did not maintain the position of the fracture fragments and allowed gross mobility. The intraoral appliances used in the 40 monkeys in groups 2 and 3 held bony fragments in position efficiently. Tedious effort was needed to fabricate the presurgical splint, and simpler forms of interdental wiring and rapidcuring acrylic reinforcement were developed.

Our criteria for "union" or "nonunion" of the fracture were established in six weeks in the 3- and 5-year-old monkeys. Prolonged immobilization of the fractures for ten weeks did not change this result. More protracted fixation did not convert nonunion to union.

The development of the fracture model as outlined for the third group of 20 monkeys gave much more consistent results. It appeared that the method of fracture production greatly influenced the response of other factors in repair. Differences between repair phenomena in the two age groups were not demonstrable in the monkeys used in the study. 


\section{Discussion}

The results obtained in these monkey studies parallel phenomena in the clinical treatment of mandibular fractures. Technics used in group 3 show that bony union is possible without intermaxillary fixation. This principle may be used clinically in some fractures of the edentulous mandible. ${ }^{14}$

The type of surgical approach to the mandible does not appear to influence the ultimate result of repair where there is an option to surgical exposure. Major intraoral surgical procedures on the mandible and maxilla are currently being done without infection or other complications. ${ }^{15}$

Current clinical practice that maintains intermaxillary fixation beyond six weeks for fracture mobility may be reconsidered. On the basis of our investigations with these fractures in monkeys, one can speculate that, if osteogenic pattern for repair of the fracture has been lost, either through infection, improper reduction, or motion, then additional prolonged immobilization probably will not bring about a union of the fracture.

It appears, from the results with the monkeys in groups 1 and 2 that the surgical defect made through the entire mandible created a challenge to repair, which exceeded that of clinical fractures and accounted for the great number of nonunions in that series.

\section{Conclusions}

Development of a model for the study of mandibular fractures required several modifications and stages. The technics finally used in group 3 allow a predictable and reproducible model for the study of repair of fractures of the midbody of the mandible. Oral contamination of the surgical site had little effect on healing. The type of surgical approach (intraoral or extraoral) used to obtain access to the fracture site did not influence the repair process under the conditions of this study. It appeared that prolonged immobilization of mandibular fractures did not reverse a process that was destined for nonunion. Fractures would unite without intermaxillary fixation if a rigid splint were used across the fracture area. The single most important factor in producing consistent results in this study was the method of fracture production and its associated trauma. A defect cut through both the buccal and lingual cortices resulted in a greater number of nonunions to the exclusion of other influences on repair. A true fracture through the lingual cortex with a surgical defect on the buccal proved to be a satisfactory model for other studies.

\section{References}

1. Yrastorya, J.A., and Kruger, G.O.: Polyurethane Polymer in the Healing of Experimentally Fractured Mandibles, Oral Surg 16:978-984, 1963.

2. Moss, R.W.: Effect of Spacing of Bone Fragments upon Healing, abstracted, $J$ Dent Res 43:828-830, 1964.

3. Anderson, M.F., and Alling, C.C.: Subcondylar Fractures in Young Dogs, Oral Surg 19:263-268, 1965.

4. Ciani, A.: The Healing Process of Mandibular Fractures Treated with Metal Internal Fixation in Animal Experiments, Rass Int Stomat Prat 16:531-544, 1965.

5. Messer, E.J.; Hayes, D.C.; and BOYNe, P.J.: Use of Intraosseous Metal Appliances in Fixation of Mandibular Fractures, $J$ Oral Surg 25:493-502, 1967.

6. Hunsuck, E.: A Method of Intra-Oral Open Reduction of Fractured Mandibles, J Oral Surg 25:533-541, 1967.

7. Clark, H., and HaYes, T.A.: A Study of the Comparative Effects of Rigid and Semirigid Fixation on the Healing of Fractures of the Mandible in Dogs, $J$ Bone Joint Surg 45A:731-741, 1963.

8. Lighterman, I., and Farrell, J.: Mandibular Fractures Treated with Plastic Polymers, Arch Surg 87:868-878, 1963.

9. Heurlin, R.J.; Gans, B.J.; and StutevILLE, O.H.: Skeletal Changes Following Fracture Dislocation of the Mandibular Condyle in the Adult Rhesus Monkey, Oral Surg 14:1490-1500, 1961.

10. Youngquist, C.O., and Cole, T.B.: Experimental Mandibular Repair with Bone Chips, Ann Otol 75:1058-1065, 1966.

11. Boyne, P.J.: Osseous Healing after Oblique Osteotomy of the Mandibular Ramus, $J$ Oral Surg 24:125-133, 1966.

12. BoYNe, P.J.: Osseous Repair and Mandibular Growth after Subcondylar Fractures, J Oral Surg 25:300-309, 1967.

13. Hurme, V.O.: Estimation of Monkey Age by Dental Formula, Ann NY Acad Sci 85: $795,1960$.

14. SADA, O.: Self-Supporting Immobilization: A New Principle in Surgical Therapy of Mandibular Prognathism in Edentulous Jaws, J Oral Surg 24:134-144, 1966.

15. OBWEgESSER, H.L.: Simultaneous Resection and Reconstruction of Parts of the Mandible via the Intraoral Route in $\mathrm{Pa}$ tients with and without Gross Infections, Oral Surg 21:693-705, 1966. 